EPJ manuscript No.

(will be inserted by the editor)

\title{
A Realistic Solvable Model for the Coulomb Dissociation of Neutron Halo Nuclei
}

\author{
Gerhard Baur ${ }^{1}, \underline{\text { Kai Hencken }}^{2}$, and Dirk Trautmann ${ }^{2}$ \\ 1 Institut für Kernphysik (Theorie), Forschungszentrum Jülich, 52425 Jülich, Germany \\ 2 Institut für Physik, Universität Basel, Klingelbergstr. 82, 4056 Basel, Switzerland
}

Received: October 31, 2018/ Revised version: October 31, 2018

\begin{abstract}
As a model of a neutron halo nucleus we consider a neutron bound to an inert core by a zero range force. We study the breakup of this simple nucleus in the Coulomb field of a target nucleus. In the post-form DWBA (or, in our simple model CWBA ("Coulomb Wave Born Approximation")) an analytic solution for the $T$-matrix is known. We study limiting cases of this $T$-matrix. As it should be, we recover the Born approximation for weak Coulomb fields (i.e., for the relevant Coulomb parameters much smaller than 1). For strong Coulomb fields, high beam energies, and scattering to the forward region we find a result which is very similar to the Born result. It is only modified by a relative phase (close to 0 ) between the two terms and a prefactor (close to 1). A similar situation exists for bremsstrahlung emission. This formula can be related to the first order semiclassical treatment of the electromagnetic dissociation. Since our CWBA model contains the electromagnetic interaction between the core and the target nucleus to all orders, this means that higher order effects (including postacceleration effects) are small in the case of high beam energies and forward scattering. Our model also predicts a scaling behavior of the differential cross section, that is, different systems (with different binding energies, beam energies and scattering angles) show the same dependence on two variables $x$ and $y$.
\end{abstract}

PACS. 25.70.De Coulomb excitation - 25.60.-t Reactions induced by unstable nuclei - 25.60.Gc Breakup and momentum distributions

\section{Introduction}

Breakup processes in nucleus-nucleus collisions are complicated, in whatever way they are treated. They constitute at least a three-body problem, which is further complicated due to the long range Coulomb force. Exact treatments (like the Faddeev-approach) are therefore prohibitively cumbersome. On the other hand, many approximate schemes have been developed in the field of direct nuclear reactions, and these approaches have been used with considerable success [1]. In this context we wish to investigate a realistic model for the Coulomb breakup of a neutron halo nucleus. With the operation of exotic beam facilities all over the world, these reactions (previously restricted essentially to deuteron induced reactions) have come into focus again. The Coulomb breakup of these nuclei is of interest also for nuclear astrophysics, since the breakup cross section can be related to the photodissociation cross section and to radiative capture reactions relevant for nuclear astrophysics [2].

An important benefit of the present model is that it can be solved analytically in the DWBA (CWBA) approximation. Thus it constitutes an ideal "theoretical laboratory" to investigate the physics of breakup reactions, certain limiting cases and its relation to other models like the semiclassical approximation, which is mainly used in the interpretation of experiments. Especially the effect of postacceleration (to be explained in more detail below) can be studied in a unique way in this approach.

\section{Description of Theoretical Model}

We consider the breakup of a particle $a=(c+n)$ (deuteron, neutron-halo nucleus) consisting of a loosely bound neutral particle $n$ and the core $c$ (with charge $Z_{c}$ ) in the Coulomb field of a target nucleus with charge $Z$.

$$
a+Z \rightarrow c+n+Z \text {. }
$$

As a further simplification the $a=(c+n)$ system is assumed to be bound by a zero range force. The bound-state wave function of the system is given by

$$
\phi_{0}=\sqrt{\frac{\kappa}{2 \pi}} \frac{\exp (-\kappa r)}{r},
$$

where the quantity $\kappa$ is related to the binding energy $E_{\text {bind }}$ of the system by

$$
E_{\text {bind }}=\frac{\hbar^{2} \kappa^{2}}{2 \mu}, \quad \mu=\frac{m_{n} m_{c}}{m_{n}+m_{c}} .
$$


In the post-form CWBA the T-matrix for the reaction Eq. (1) can be written as [3]

$$
\begin{aligned}
T & =\left\langle\chi_{\mathbf{q}_{c}}^{(-)} \psi_{\mathbf{q}_{n}}\left|V_{n c}\right| \chi_{\mathbf{q}_{a}}^{(+)} \phi_{0}\right\rangle \\
& =D_{0} \int d^{3} R \chi_{\mathbf{q}_{c}}^{(-)}(\mathbf{R}) e^{-i \mathbf{q}_{n} \cdot \mathbf{R}} \chi_{\mathbf{q}_{a}}^{(+)}(\mathbf{R}),
\end{aligned}
$$

with the "zero range constant" $D_{0}$ given by $D_{0}=$ $\frac{\hbar^{2}}{2 \mu} \sqrt{8 \pi \kappa}$. The initial state is given by the incoming Coulomb wave function $\chi_{\mathbf{q}_{a}}^{(+)}$with momentum $\mathbf{q}_{a}$ and the halo wave function $\phi_{0}$. The final state is given by the independent motion of the core described by the outgoing Coulomb wave function $\chi_{\mathbf{q}_{c}}^{(-)}$in the Coulomb field of the target nucleus $Z$ with asymptotic momentum $\mathbf{q}_{c}$ and the free neutron with momentum $\mathbf{q}_{n}$, described by a plane wave. In these wave functions the Coulomb interaction is taken into account correctly to all orders. In our model there is no resonance structure in the $c+n$ continuum. This is clearly a good assumption for the deuteron and also for other neutron halo systems.

There exists another form of the $T$-matrix element, which is not equivalent to Eq. (5). It is called the "priorform" [1]. The final state is described by a c.m. motion of the $(c+n)$ system (as a Coulomb wave function) and a relative wave function of the unbound $(c+n)$ system.

The present "post-form" description, Eqs. (4), (5) includes the effects of "postacceleration". "Postacceleration" arises in a purely classical picture of the breakup process. This is nicely discussed in [4] (We show their figure 5 here as our Fig. 11). The nucleus $a=(c+n)$ moves up the Coulomb potential, loosing the appropriate amount of kinetic energy. At the "breakup point" (marked as "breakup occurs here", see Fig 11), this kinetic energy (minus the binding energy) is supposed to be shared among the fragments according to their mass ratio (assuming that the velocities of $c$ and $n$ are equal). Running down the Coulomb barrier, the charged particle $c$ alone (and not the neutron) gains back the Coulomb energy, resulting in its "postacceleration". Of course this picture is based on the purely classical interpretation of this process, and will be modified in a quantal treatment, where such a "breakup point" does not exist. The correct semiclassical limit of the theory in this case can be found, e.g., in [5]. A purely classical formula for this postacceleration, where the "breakup point" corresponds to the distance of closest approach i.e., $b=r$ in Fig. 1 - is given in [6]. Postacceleration is clearly observed in low energy deuteron breakup, in the (fully quantal) theoretical calculations as well as in the corresponding experiments, see Fig. 2 and also, e.g., [7, 8].

The formula Eq. (禹) is also useful for the description of the Coulomb dissociation of halo nuclei at high beam energies, see 10. Within this theory postacceleration effects become negligibly small in the high energy region. This is seen in the numerical calculations [10] and in the analytical investigations to be described below. It can, e.g., be applied to ${ }^{11} \mathrm{Be}$ and ${ }^{19} \mathrm{C}$ Coulomb dissociation experiments [11,12] (We disregard here the importance of finite range effects).

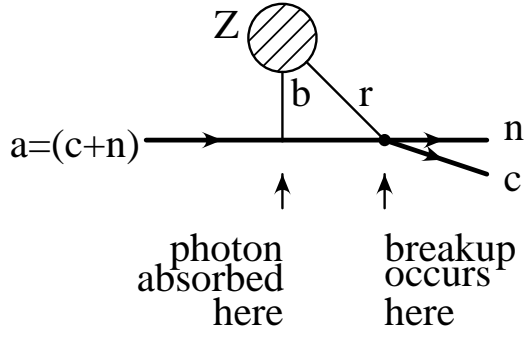

Fig. 1. A schematic view of Coulomb-breakup, as adapted from 4 . The distance from the target nucleus to the breakup point is denoted by $r$.

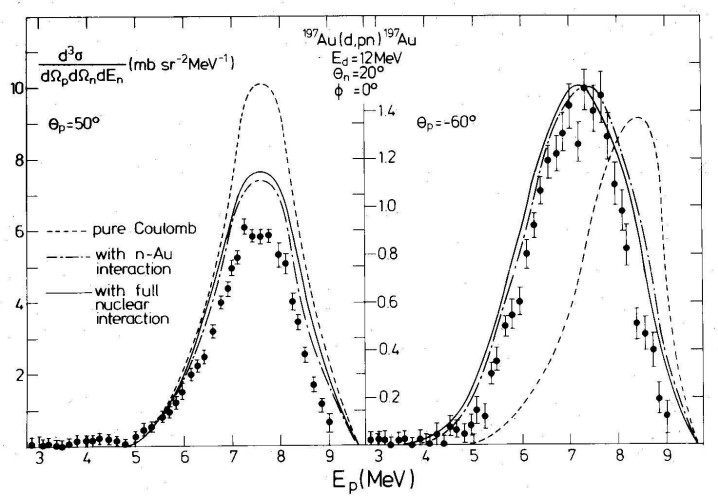

Fig. 2. Comparison of calculations and measurement for the deuteron breakup coincidence cross section on ${ }^{197} \mathrm{Au}$ at $E_{d}=$ $12 \mathrm{MeV}$ (Fig. 4 of [8]). The postacceleration effect can clearly be seen, as the maximum of the proton energy $(\sim 7.5 \mathrm{MeV})$ is larger than the one of the neutron $(\sim 2.5 \mathrm{MeV})$. The experimental data are taken from [9].

On the other hand the $1^{\text {st }}$ order semiclassical Coulomb excitation theory was widely applied in the past years to the Coulomb dissociation of high energy neutron halo nuclei, see, e.g., [13]. The theory corresponds to the "prior form", mentioned above. The question of higher order electromagnetic effects was studied recently in [14] within this framework. These effects were found to be small, for zero range as well as finite range wave functions of the $a=(c+n)$-system. It seems interesting to note that postacceleration effects arises through higher order electromagnetic effects in straight line semiclassical theories, see 15. Through the interference of $1^{\text {st }}$ and $2^{\text {nd }}$ order amplitudes even a "post-deceleration" can arise, as was seen in that paper.

In this work we want to establish the relation between the apparently very different post-form CWBA and semiclassical theory. It was recently noticed 13 that in the limit of Coulomb parameters $\eta_{a}=Z_{c} Z e^{2} / \hbar v_{a} \ll 1$ (i.e. in the Born approximation), where $v_{a}$ denotes the velocity of particle $a\left(v_{a}=\frac{\hbar q_{a}}{m_{a}}\right)$, both theories give the same result. Expanding the Coulomb wave functions up to first order in the Coulomb fields one finds 

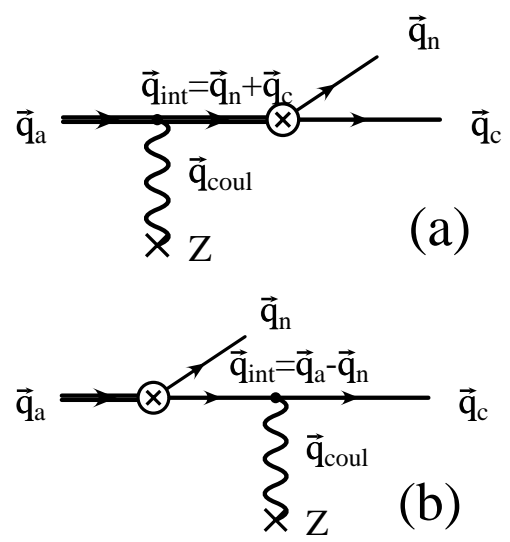

Fig. 3. The two bremsstrahlung type of graphs, which describe the Coulomb breakup in the Born approximation. Threemomentum conservation at each vertex determines the intermediate momenta $\mathbf{q}_{\text {int }}$.

$$
\begin{aligned}
T_{\text {Born }}= & f_{\text {coul }} D_{0} \times \\
& {\left[\frac{1}{q_{a}^{2}-\left[\mathbf{q}_{c}+\mathbf{q}_{n}\right]^{2}}+\frac{m_{n}}{m_{a}\left[q_{c}^{2}-\left(\mathbf{q}_{n}-\mathbf{q}_{a}\right)^{2}\right]}\right] . }
\end{aligned}
$$

Here $f_{\text {coul }}=2 \eta_{a} q_{a} /\left(\mathbf{q}_{\text {coul }}\right)^{2}$ is the usual Coulomb amplitude with the "Coulomb push" $\mathbf{q}_{\text {coul }}=\mathbf{q}_{a}-\left(\mathbf{q}_{c}+\mathbf{q}_{n}\right)$, for further details see [3]. The two terms in the parenthesis correspond to the two graphs shown in Fig. 3. For small values of $q_{\text {coul }}$ the two terms almost cancel and the expansion in $q_{\text {coul }}$ was found to be in agreement with the semiclassical result, see [13] and below.

We now show that this agreement is also true in the case of arbitrary values of $\eta_{a}$ and $\eta_{c}$. The beam energy must be high (compared to the binding energy $E_{\text {bind }}$ ) and the two fragment need to be scattered into forward angles.

This is reminiscent of the result in the theory of bremsstrahlung. Replacing the neutron by a photon the diagrams of Fig. 3 are identical to the bremsstrahlung in lowest order. In this case it was already noticed that the Born result remains valid for arbitrary values of $\eta$ for high energies and small scattering angles 16 . Here we want to show that the same applies in this case.

The $T$-matrix can be evaluated analytically in this model due to well known Nordsieck formula [17], see Eqs. (11)-(13) of [3]. Using this formula one obtains the $T$-matrix Eq. (4) in terms of a hypergeometric function $F$ as well as its derivative $F^{\prime}$. The argument of the hypergeometric function $F$ (and $F^{\prime}$ ) is given by [3, 10]:

$$
\begin{aligned}
& \zeta(\lambda)= \\
& \frac{2 q_{\mathrm{coul}}^{2}\left(q_{a} q_{c}+\mathbf{q}_{a} \mathbf{q}_{c}\right)-4\left(\mathbf{q}_{\mathrm{coul}} \mathbf{q}_{a}+\lambda q_{a}\right)\left(\mathbf{q}_{\mathrm{coul}} \mathbf{q}_{c}-\lambda q_{c}\right)}{\left(q_{\mathrm{coul}}^{2}-2 \mathbf{q}_{\mathrm{coul}} \mathbf{q}_{a}-2 \lambda q_{a}\right)\left(q_{\mathrm{coul}}^{2}+2 \mathbf{q}_{\mathrm{coul}} \mathbf{q}_{c}-2 \lambda q_{c}\right)} .
\end{aligned}
$$

We observe that (for $\lambda=0$ ) this parameter $\zeta(0)$ is found to be negative and $-\zeta(0) \gg 1$ for beam energy large compared to the binding energy and for perpendicular momentum transfers $q_{\perp} \gg 2 \eta_{a} q_{\|}$(nonadiabatic case), where $q_{\|}=\omega / v$ with $\hbar \omega=E_{\text {bind }}+E_{\text {rel }}$ and where the relative energy between $c$ and $n$ is $E_{\text {rel }}=\frac{\hbar^{2} q^{2}}{2 \mu}$ with the relative momentum given by $\mathbf{q}=\frac{m_{c} \mathbf{q}_{n}-n_{n} \mathbf{q}_{c}}{m_{a}}$. It was already noticed in the numerical evaluation of the process that, due to $-\zeta(0) \gg 1$ that the hypergeometric series does not converge and an analytic continuation had to be used. Here we use this fact to our advantage and make a linear transformation to get the argument of the hypergeometric function close to 0 . The transformation we are using leads to the argument of the hypergeometric function $z=\frac{1}{1-\zeta(0)}$ (Eq. 15.3.7 of [18). In this respect our approach differs from the one used in the bremsstrahlung case, where a transformation giving an argument close to one is used. Using only the lowest order term in the hypergeometric series one obtains after some algebra (up to an overall phase)

$$
\begin{aligned}
T \approx & 4 \pi D_{0} f_{\text {coul }} e^{-\frac{\pi}{2} \xi}\left[e^{-i \phi} \frac{1}{q_{a}^{2}-\left(\mathbf{q}_{n}+\mathbf{q}_{c}\right)^{2}}\right. \\
& \left.+e^{+i \phi} \frac{m_{c}}{m_{a}} \frac{1}{q_{c}^{2}-\left(\mathbf{q}_{n}-\mathbf{q}_{a}\right)^{2}}\right]
\end{aligned}
$$

Hereby, the relative phase is $\phi=\sigma_{0}\left(\eta_{c}\right)-\sigma_{0}\left(\eta_{a}\right)-\sigma_{0}(\xi)-$ $\xi / 2 \log |\zeta(0)|$. The $\sigma_{0}(\eta)=\arg \Gamma(1+i \eta)$ are the usual Coulomb phase shifts, and $\xi=\eta_{c}-\eta_{a}$. The correspondence to the Born result is clearly seen. One only has an additional prefactor $e^{-\frac{\pi}{2} \xi}$ and a relative phase $e^{ \pm i \phi}$ between the two terms. The phase $\phi$ obviously is $O(\xi)$. Since $v_{c} \sim v_{a}$ the quantity $\xi$ is usually very small and so is $\phi$ for the cases of $[11,12$. The prefactor is also well known in the semiclassical theory, where it accounts for the replacement of the "Coulomb bended" trajectories with the straight line trajectories. Both corrections vanish in the limit $\xi \rightarrow 0$ and the result coincides with the usual Born approximation (even if $\eta_{a}$ and $\eta_{c}$ are not small).

We have seen that the $T$-matrix in the case of large Coulomb parameters $\eta_{a}$ and $\eta_{c}$ corresponds to the Born result (small Coulomb parameter) in the sudden (or nonadiabatic) case $q_{\perp} \gg 2 \eta_{a} q_{\|}$. We note that the derivation of Eq. (8) only depends on the condition $-\zeta(0) \gg 1$ (and not on the values of the $\eta$ 's). For $\eta_{a}, \eta_{c} \gg 1$ one can define a classical path for both $a$ and $c$ in the initial and final state and Eq. (8) can be related to the semiclassical approach (see the discussion below following Eq. (9)). We expect to find a connection between the semiclassical theory and the adiabatic case $\left(q_{\perp}<2 \eta_{a} q_{\|}\right)$and the fully quantal expression for the $T$-matrix. For the adiabatic regime the well known exponential decrease with the adiabacity parameter is observed in the numerical calculations. In this case, the inequality $-\zeta(0) \gg 1$ is not generally satisfied. We are presently working to see how the semiclassical limit can be obtained with analytical methods in this case also. Such a method would also be valid in both the adiabatic and nonadiabatic case as long as $\eta_{a}, \eta_{c} \gg 1$.

A similar situation is encountered in the theory of bremsstrahlung and Coulomb excitation, see Section II E of [19]. There a fully quantal expression for the differential cross-section for dipole Coulomb excitation is given in II E.62. It looks similar to the corresponding expression 
for Coulomb breakup (see [3]). The semiclassical "variant" of this formula is found in II E.57. It is noted there that it can be obtained from the quantal expression by letting at the same time $\eta_{a}$ and $\eta_{c}$ go to infinity and perform a confluence in the hypergeometric functions.

\section{Scaling Properties}

In many experimental situations the Coulomb push $\mathbf{q}_{\text {coul }}$ is small. Having found that the full CWBA results agrees with the Born result for small scattering angles, we can expand Eq. (6) or Eq. (8) with $\phi=\xi=0$ for small values of $q_{\text {coul }}$. We obtain

$$
T=f_{\text {coul }} \frac{2 D_{0}}{\pi^{2}} \frac{m_{n}^{2} m_{c}}{m_{a}^{3}} \frac{2 \mathbf{q} \cdot \mathbf{q}_{\mathrm{coul}}}{\left(\kappa^{2}+q^{2}\right)^{2}} .
$$

This result is in remarkable agreement with the usual $1^{\text {st }}$ order treatment of electromagnetic excitation in the semiclassical approximation.

In the semiclassical approach the scattering amplitude is given by the elastic scattering (Rutherford) amplitude times an excitation amplitude $a(b)$, where the impact parameter is related to the $q_{\perp}$ and $\eta$, see above. The absolute square of $a(b)$ gives the breakup probability $P(b)$, in lowest order (LO). It is given by 13, 14

$$
\frac{d P_{\mathrm{LO}}}{d q}=\frac{16 y^{2}}{3 \pi \kappa} \frac{x^{4}}{\left(1+x^{2}\right)^{4}}
$$

where the variable $x$ is related to the relative momentum between $n$ and $c$ by $x=\frac{q}{\kappa}$ and $y$ is a strength parameter given by

$$
y=\frac{2 Z Z_{c} m_{n} e^{2}}{\hbar v_{a} m_{a} b \kappa} .
$$

This formula shows very interesting scaling properties: Very many experiments, for neutron halo nuclei with different binding energy, beam energy, scattering angles (or $\mathbf{q}_{n}$ and $\mathbf{q}_{c}$ ) all lie on the same universal curve! (Corrections for finite values of $\xi_{\text {eff }}=\omega b / v=\xi(\theta)=2 \eta_{a} q_{\|} / q_{\perp}$ should also be applied, according to [14].) It will be interesting to see in future calculations under what conditions (beam energy, ...) one finds deviations from this simple scaling behavior. E.g., postacceleration effects will lead to such scaling violations.

\section{Conclusion and Outlook}

The present model can be seen as a "theoretical laboratory", which allows to study analytically, as well as, numerically the relation between quantal and semiclassical theories, and the importance of postacceleration effects. We mention that from an experimental point of view, the postacceleration effects are not fully clarified, see, e.g., [12, 20] ("postacceleration") and on the other hand [21] ("no postacceleration"). Finally, let us mention recent work on the electromagnetic dissociation of unstable neutron-rich oxygen isotopes 22, 23]. These authors deduce photoneutron cross-sections from their dissociation measurements. If the neutrons are emitted in a slow evaporation process in a later stage of the reaction, the question of postacceleration is not there. On the other hand, for the light nuclei there is some direct neutron emission component and the present kind of theoretical analysis further proves the validity of the semiclassical approach used in 22].

Postacceleration effects are also of importance for the use of Coulomb dissociation for the study of radiative capture reactions of astrophysical interest. We expect that our present investigations will shed light on questions of postacceleration and higher order effects in these cases also.

\section{References}

1. N. Austern, Direct Reaction Theory (Wiley, New York, 1970).

2. G. Baur and H. Rebel, Ann. Rev. Nucl. Part. Sci. 46 (1996) 321.

3. G. Baur and D. Trautmann, Nucl. Phys. A191 (1972) 321.

4. A. Galonsky et al., Preprint MSUCL-951, October 1994, available from www.nscl.msu.edu.

5. G. Baur, M. Pauli, and D. Trautmann, Nucl. Phys. A224 (1974) 477.

6. G. Baur, C. A. Bertulani, and D. M. Kalassa, Nucl. Phys. A550 (1995) 107.

7. G. Baur and D. Trautmann, Phys. Reports 25C (1976) 293.

8. G. Baur, F. Roesel, D. Trautmann, and R. Shyam, Phys. Reports 111 (1984) 333.

9. L.Jarczyk et al., Phys. Lett. 39B (1972) 191.

10. R. Shyam, P. Banerjee, and G. Baur, Nucl. Phys. A540 (1992) 341.

11. T. Nakamura et al., Phys. Rev. Lett. 83 (1999) 1112.

12. T. Nakamura, Phys. Lett. B331 (1994) 296.

13. G. Baur, K. Hencken, D. Trautmann, S. Typel, and H.H. Wolter, Proc. of the Int. Workshop, Erice 16-24 September, Prog. in Part. and Nucl. Phys. 46 (2001) 99, also available as nucl-th/0011061.

14. S. Typel and G. Baur, Phys. Rev. C64 (2001)024601, also available as nucl-th/0101033.

15. S. Typel and G. Baur, Nucl. Phys. A573 (1994) 486.

16. L. D. Landau and E. M. Lifschitz, Lehrbuch der theoretischen Physik IV, Quantenelektrodynamik (Akademie Verlag, Berlin, 1986)

17. A. Nordsieck, Phys. Rev. 93 (1954) 785.

18. M. Abramowitz and I.A. Stegun, Handbook of Mathematical Functions (National Bureau of Standards, Washington, DC, 1964).

19. K. Alder, A. Bohr, T. Huus, B. Mottelson, and A. Winther, Rev. Mod. Phys. 28 (1956) 432.

20. K. Ieki et al., Phys. Rev. Lett. 86 (2001) 730.

21. J. E. Bush et al., Phys. Rev. Lett. 81 (1998) 61.

22. A. Leistenschneider et al., Phys. Rev. Lett. 86

23. T. Aumann, this conference. 